\title{
Linguistic influences on visual memory
}

\author{
JOHN L. SANTA and LINDA BAKER \\ Rutgers University, Douglass College, New Brunswick, New Jersey 08903
}

\begin{abstract}
The Whorf-Sapir hypothesis has raised considerable controversy in the literatures of psychology and anthropology. Several misconceptions of the hypothesis are reviewed, and the hypothesis was experimentally supported in a visual reproduction paradigm. Subjects were first given label training for a set of figures, and were then asked to recall by drawing the shapes. Training with categorized labels resulted in a $25 \%$ improvement in recall when compared to a condition with nonword (paralog) labels. Even stronger evidence of linguistic influence on visual memory was obtained by examining the order of recall. The conceptual relationships among labels strongly influenced the sequence of reproductions.
\end{abstract}

Does language in any way influence the memory of visual stimuli? This question is directly related to the Whorf-Sapir (Whorfian) hypothesis which suggests that language is a powerful determinant of perception and thought. The hypothesis has never been clearly or singularly expressed, and so exists in several versions. A common distinction is made between "weak" and "strong" forms of the Whorfian hypothesis (Funkhouser, 1968; Slobin, 1971). The strong form, which holds that aspects of language can determine thought, is routinely rejected by experimental psychologists. The weak form suggests that "One is not fully a prisoner of one's language" but that language "can predispose people to think or act in one way rather than another" (Slobin, 1971, p. 122).

Actually, the distinction is quite misleading, and probably stands as an excellent example of the Whorfian hypothesis in action. Psychology has, for the most part, adopted a probabilistic view of behavior; consequently, to say that language merely influences behavior is only to assert a scientific axiom. There is nothing necessarily "weak" about a variable which influences but does not strictly determine behavior, since influence is the strongest form of causality allowed to our science. It is interesting that labeling the hypothesis as "weak" seems to have led to a ready acceptance, but also a consequent devaluation of its importance, e.g., Slobin, 1971.

While it is not reasonable for psychologists to distinguish the strong and weak forms of the Whorfian hypothesis, it is possible to consider the strength and extent of the linguistic influence. Investigations of linguistic influence on our sensory-perceptual experience are few and their findings are not compelling. Lenneberg $(1967$, p. 348$)$ reports virtually no effect of different linguistic conventions on the ability to discriminate color hues. More promising support for the hypothesis

Requests for reprints should be addressed to John L. Santa, Department of Psychology, Rutgers University, Douglass College, New Brunswick, New Jersey 08903. The research was in part supported by Rutgers Research Council and Biomedical grants to the first author. We would like to express oux appreciation to Matthew H. Erdelyi and Edith Neimark for their help ful com ments on an earlier draft of this article. comes from studies of linguistic influences on memory of nonverbal stimuli. A series of experiments has shown a correlation between verbal codability and visual memory (Brown \& Lenneberg, 1954; Lantz \& Stefflre, 1964), but such correlational data are weak in the sense of not necessarily implying causality (Rosch, 1973).

The Whorfian hypothesis is better supported by the literature which documents the influence of labeling on memory of visual forms (Ellis, 1968; Ellis \& Muller, 1964; Santa \& Ranken, 1968, 1972). These studies show that providing subjects with unique label training for a set of nonsense shapes can improve recognition of the shapes at least when the stimulus is complicated (Ellis \& Muller, 1964), or when there is a large number of stimuli to be maintained (Santa \& Ranken, 1972). The effects on recognition are strongest when the labels are in some way representative of the visual stimuli (Ellis, 1968). Scveral experiments have also shown effects of labeling with longer retention intervals (Daniel \& Ellis, 1972; Ellis \& Daniel, 1971), although Santa and Ranken (1972), in a somewhat different paradigm, were unable to obtain labeling effects beyond a short-term memory range.

Ellis (Ellis, 1972; Ellis \& Muller, 1964) has explained the effects of label training in recognition tasks on the basis of a conceptual coding hypothesis which suggests that label training influences memory primarily by focusing attention to distinctive stimulus features. If the label is not representative of the stimulus, it fails to focus attention and, consequently, has little effect on recognition memory. Such effects of labeling are certainly in accord with the Whorfian hypothesis, but do not capture the capricious influence of language which the hypothesis implies. The Whorfian hypothesis suggests that a name can influence our visual memories, even if applied arbitrarily, without reference to the stimulus features.

Effects of arbitrary labels are not usual in the recognition paradigm (Ellis, 1968, 1972), but the might be evident in a variety of other situations. In particular, Santa (Santa, \& Ranken, 1972; Santa, Reference Note 4) has suggested that names in general, even arbitrary ones, 
might influence the retrieval process. Thus, it might be possible to obtain broader support for the Whorfian hypothesis in a task requiring recall or reproduction of visual stimuli. The reproduction task presumably places more emphasis on the retrieval process than does a recognition paradigm, and consequently offers an extra source of potential linguistic influence.

The influence of verbal codes on recall of visual stimuli, as.opposed to recognition, has been explored in at least two paradigms, neither of which has been decisive thus far. In one approach, subjects are presented with readily labeled pictorial stimuli and are asked to recall the names of these stimuli (Frost, 1971, 1972; Funkhouser, 1968). These experiments show that subjects' recall of object names is organized by conceptual class so that the names of objects belonging to a similar conceptual category are recalled in close proximity. Inferences from these data to the influence of language on memory of visual stimuli are somewhat clouded by the fact that the stimuli were readily encoded by names and that subjects are only required to recall the verbal labels rather than reproduce the visual stimuli. The problem of requiring label recall of visual stimuli is documented by Tversky's (1974) finding that subjects, given a stimulus consisting of an object picture and its name, spend most of their time observing (and rehearsing) the name if they expect the criterion task to be one of name recall. Thus, when subjects know the task requires label recall, they might fail to attend to the visual aspects of the stimulus. Clustering of the labels does not necessarily imply an influence on visual memory.

The second approach to recall of visual stimuli is embodied in the much older literature on memory of form inspired by Gestalt psychology. The Carmichael, Hogan, and Walter (1932) experiment is prototypic of this effort. Subjects were presented with ambiguous line drawings accompanied by labels which resolved the ambiguity of the figures in one of two possible directions. For example, a figure consisting of two circles connected with a straight line was labeled either "barbell" or "eyeglasses." It was found that subjects' reproductions of the stimuli were biased in the direction suggested by the referents of the attached labels. Although these data suggest an effect of language on visual memory, the possibility of response bias has not been ruled out during the subsequent 40 years of related research. The response bias interpretation suggests that the label might not influence the memory of the visual form, but only influences the criterion for drawing particular aspects of the figure. Despite this ambiguity, the memory of form literature is perhaps the best evidence to date that language can influence the representation of visual stimuli.

The present experiment follows from the memory of form tradition, attempting to demonstrate an influence of verbal coding on visual memory by examining subjects' ability to reproduce visual material. However, while the Gestalt literature focused on the effect of verbal suggestion on the individual representation, the present work examines the influence of language on the organization and retrieval of an entire set of representations. To examine the order of reproduction offers a more sensitive index of linguistic influence. One might expect a stronger effect of language when considering the organization of output than when measuring details of an individual reproduction. The experiment is relevant to the Whorfian hypothesis in that it attempts to show that linguistic habits can affect nonverbal memories.

The paradigm consisted of giving subjects label training to establish associations between words and a set of nonsense shapes. The words were arbitrarily assigned to shapes, and so were minimally representative. However, the words themselves were highly related in that they were members of several common semantic categories. A control group was trained with paralog (nonsense) names. After training, subjects were required to reproduce as many of the shapes as possible in a free recall task. The basic expectation was that the categorized labels would exert a strong influence on both the likelihood and order of reproduction. If subjects are guided by language in their retrieval of visual information, then the shapes should exhibit a high degree of output clustering by semantic category. The paralog control should recall fewer items and should not display the same organization of reproduction.

\section{METHOD}

\section{Subjects}

Thirty-seven undergraduates enrolled in Douglass College psychology courses served as subjects. Because the subjects were not told in advance that there would be a second recall task after a 1-week interval, some subjects (six) were not available for the retest. Their data were discarded and the results reported are based on the complete data of 31 subjects.

\section{Stimulus Materials}

The stimuli consisted of 16 nonsense shapes paired with verbal labels which were either names of categorized objects or paralogs (see Figure 1). The nonsense shapes were simple outline drawings, constructed by combining parts of geometric forms. There were no strict rules of construction, but the shapes were designed with two major considerations: there should be as few structural similarities among the shapes as possible, and the shapes should bear as little resemblance to real objects as possible. These restrictions were imposed in order to minimize subjects' clustering their recall according to similarity of visual features, and to minimize the likelihood of subjects' generating their own labels. The experimenter-supplied labels were 16 words belonging to four categories (fruits, animals, musical instruments, and furniture) selected from the five most frequent items for each category in the Battig and Montague (1969) category norms. Sixteen paralogs, two-syllable nonsense words, were chosen from a list prepared by Noble (1952) on the basis of their dissimilarity to real words and their high degree of pronunciability.

The stimuli were made into slides and were seen as white 
outlines and letters against a black background. The sequences were videotaped and presented to subjects on an 11 -in. television monitor.

\section{Design}

The experiment consisted of a 3 by 2 factorial design with three name training conditions and two retention intervals. All subjects were tested at both retention intervals (immediate and 1-week delay). The naming conditions differed with respect to the type of label used for each shape. In the first condition, Category Names I (CI), each of the 16 nonsense shapes was randomly paired with one of the category names. The same verbal labels were used for the second condition, Category Names II (CII), but they were orthogonally paired with different shapes so that only one shape from a particular category in CI remained in the same category in CII, and the label was changed for the one common shape within that category. Condition CII, included as a replication control, was expected to attain the same recall level as $\mathrm{CI}$. Condition CII also provides a powerful test of the hypothesis that the labels will determine the organization of subjects' reproductions. The recall output of CI and CII should be organized quite differently in terms of shape reproduction, since label-shape assignments were orthogonal by category for the two conditions. Paralog names were randomly assigned to shapes for the third condition (P). The paralog condition provides a baseline index of clustering, and in many ways is a better control than either an unnamed-observation group, or a condition trained with meaningful but uncategorized labels. Paralog training is quite similar to category label training, and has the advantage of reducing spontaneous labeling when compared to an unnamed condition (Santa, 1969). The paralog condition also provides an excellent control for shape clustering in the absence of a semantic influence of real words. A group trained with meaningful, uncategorized labels would be useful to assess the importance of categorical vs. meaningful labels on total reproduction, but the present experiment was primarily concerned with linguistic influences on memory organization. In a related experiment, Nicosia (Reference Note 2) has demonstrated that categorized label training leads to more correct reproductions than training with uncategorized labels, which in turn are better than no label training.

\section{Procedure}

The experiment consisted of five parts: (a) label-learning task, (b) paired-associate training task, (c) immediate free recall of the shapes, (d) delayed (1-week) free recall of the shapes, and (e) delayed (1-week) free recall of the labels. 1

The first part of the experiment was a free recall list-learning task. Subjects were given three trials in which to learn the 16 words which were later used as labels for their particular condition. Subjects in the category name conditions were told that the words belonged to four conceptual categories, but they were not specifically told to categorize the words in recall. All groups were told to recall the words in any order they wished.

The lists were presented to subjects in Conditions CI and CII

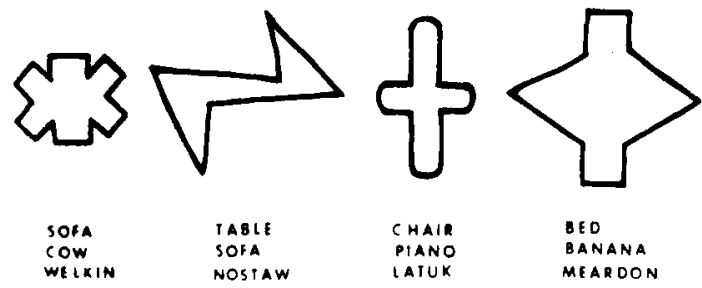

Figure 1. A sample of the stimulus shapes together with labels for the three name training conditions (Row 1-CI, Row 2-CII, Row 3-paralog).
Table 1

Mean Number of Correct Responses by Condition for Final Two Training Trials

\begin{tabular}{cccc}
\hline & CI & CII & P \\
\hline Trial 9 & 15.00 & 14.83 & 14.22 \\
Trial 10 & 15.56 & $15.1 ?$ & 14.44 \\
\hline
\end{tabular}

by means of a tape recorder; the paralog list was presented visually with slides to enable subjects to learn the graphemic representation of the words. The words were presented in random orders to ConditionsCI and $\mathrm{CII}$ at $2-\mathrm{sec}$ intervals, and for the $P$ condition, the projector was set to advance at 5 -sec intervals. A $90-\mathrm{sec}$ recall period followed each trial.

Instructions were then given for the paired-associate training task. The 16 label-shape pairs were first presented singly on videotape for two seconds to familiarize subjects with the stimuli. Ten training trials were administered, each consisting of a random permutation of the 16 label-shape pairs. For each stimulus pair, the label was first presented alone for $7 \mathrm{sec}$. At this time, the subject was to anticipate which shape was paired with that label and draw the shape in her/his response booklet. A signal indicated the onset of the associated nonsense shape which was presented next to the label for $2 \mathrm{sec}$. There was a $2-\mathrm{sec}$ interval before the next label was presented.

Immediately after the last training trial, subjects were instructed to recall as many shapes as they could and to draw them in their response booklets. They were specifically told not to include the associated labels. A 5-min time limit was imposed.

The delayed retention interval was as close to 1 week in length as possible. (CI: $\overline{\mathrm{X}}=6.56$ days; $\mathrm{CII}: \overline{\mathrm{X}}=6.42$ days; $\mathrm{P}$ : $\bar{X}=6.30$ days.) Recall instructions were identical to those used for the immediate recall test, and subjects were given 5 min to draw the shapes. Upon completion of the task, subjects were instructed to recall the labels. They were given $90 \mathrm{sec}$ to write their responses on a separate sheet of paper.

\section{RESULTS}

\section{Training}

The mean number of words recalled on the final trial of the list-learning task were $15.11,14.83$, and 9.5 for Conditions $\mathrm{Cl}$, CII, and $\mathrm{P}$, respectively. An analysis of variance indicated that the difference between conditions was significant, $F(2,28)=16.06 ; p<.001$. The difference obviously is due to the poor label recall of the paralog condition.

The paired-associate training data were scored for the number of shapes which were correctly paired with the labels. The mean number of correct responses made by subjects in the three training conditions on Trials 9 and 10 is shown in Table 1. An unweighted-means analysis of variance indicated that the effect of naming condition was not significant, $F<1.0$, and suggests that all subjects learned the label-shape pairings equally well by the end of training. Subjects' performance improved over trials, as shown by an $F(1,27)$ of $4.20 ; p<.05$. There was no interaction between naming condition and training trials, $\mathrm{F}<1.0$.

\section{Recall Performance}

The mean number of shapes recalled by subjects in 
Table 2

Mean Number of Shapes Recalled by Condition for Two Recall Tests

\begin{tabular}{lrrr}
\hline & \multicolumn{1}{c}{ CI } & \multicolumn{1}{c}{ CII } & \multicolumn{1}{c}{$\mathrm{P}$} \\
\hline Immediate Recall & 15.56 & 15.08 & 12.10 \\
$\quad$ Incorrect Shapes & .44 & .67 & 1.50 \\
Delayed Recall & 14.44 & 13.67 & 10.00 \\
$\quad$ Incorrect Shapes & .56 & 1.00 & 1.80 \\
\hline
\end{tabular}

the three conditions for the two recall tests is presented in Table 2. Only those reproductions which possessed all of the critical features of the original stimuli were counted as correct recalls. An independent scoring of a partial set yielded a $94 \%$ interrater agreement. An unweighted-means analysis of variance was performed on the total number of shapes recalled. A significant main effect was obtained for naming condition, $F(2,28)=15.37 ; p<.001$. Subtests performed on the naming factor revealed that subjects in Condition $P$ recalled fewer shapes than the worst of the category conditions $(\mathrm{CII}), \mathrm{F}(1,28)=16.80 ; \mathrm{p}<.001$; and that the difference between $\mathrm{CI}$ and $\mathrm{CII}$ was not reliable, $\mathrm{F}<1.0$. This effect of category labels is really quite substantial, producing approximately a $25 \%$ difference in recall between the category and paralog label conditions. The main effect of retention interval was also significant, $F(1,28)=16.80 ; p<.001$, indicating that performance was generally worse on the delayed test. The interaction of Naming Condition by Retention Interval was not significant, $F(2,28)=1.11 ; p<.30$. suggesting that naming condition did not influence the decrease in recall over trials.

The recall protocols were also scored for the number of incorrect shapes, those shapes which were not included in the recall totals because they were incomplete or inaccurate. The mean number of incorrect shapes drawn during the two recall trials is also shown by condition in Table 2 . Analysis of these data revealed a marginal effect of naming condition, $F(2,28)=3.14$; $p<.10$, but no other reliable effects. This may suggest that meaningful labels improve the accuracy of individual reproductions in addition to increasing the number of shapes recalled. These data also indicate that subjects in the paralog condition are somewhat less conservative in their reproduction responses, and tend to discredit a criterion explanation of total reproduction differences.

The mean number of labels recalled at the delayed-test session were $15.44,14.58$, and 3.20 for Conditions $\mathrm{CI}, \mathrm{CII}$, and $\mathrm{P}$, respectively, $F(2,28)=136.15 ; p<.001$. Again, the difference is clearly due to the poor label recall of the paralog condition.2

\section{Clustering Measures}

Clustering scores were obtained by counting the number of category repetitions and then calculating a corrected ratio of repetitions (CRR) (Frost, 1971). A repetition was defined as any two shapes from the same label category appearing consecutively in free recall. Incorrect shapes were excluded, and shapes appearing before or after intrusions were not considered to be repetitions. The CRR measure converts each subject's repetition score into a restricted range $(0.0-1.0)$ as follows: (number of repetitions recalled)/[(number of words recalled) - (number of categories recalled)]

Two different scoring systems were used to count category repetitions. First, the data were scored as though subjects in all conditions had been trained with the label-shape pairs used by Condition CI. For example, the four shapes in Figure 1 are paired with furniture labels in $\mathrm{CI}$; with labels belonging to four different categories in CII; and with four paralogs in P. Using the $\mathrm{Cl}$ scoring system, these shapes would be scored as members of a single category (1), regardless of subjects' naming condition. The second scoring system treated all subjects as if they had been trained in Condition CII. Referring again to Figure 1, the shapes would be scored as members of Categories 1, 2, 3, and 4 for all subjects, indicating that each shape belongs to a different category on the basis of Scoring System CII.

Two CRRs were calculated for each subject, based on the number of repetitions obtained using the $\mathrm{CI}$ and $\mathrm{CII}$ scoring systems. Since the CRR measure is restricted to a 0.0-1.0 range, the scores were transformed by the arcsine, and then they are analyzed with an unweighted-means analysis of variance. The analysis was performed on a three-factor design of Naming Condition by Scoring Method by Test. The test factor (immediate vs. delayed) had no reliable effect on clustering scores, $\mathrm{F}<1.0$, nor did it interact with any of the other factors. The main effect of naming condition was significant, $F(2,28)=6.95, p<.01$, and indicates that the paralog condition exhibited less clustering than either of the category name conditions.

Figure 2 shows the data of primary interest, the interaction of Naming Condition by Scoring Method, $F(2,28)=20.91, p<.001$. The figure illustrates the strong influence of label training on the order of shape reproduction. Both category name conditions exhibited a high degree of clustering by label category when scored by their respective methods. Such clustering of the reproductions is clearly not due to a covariance of visual features with the category name assignment. The completely crossed interaction of Naming Condition by Scoring Method indicates that the order of shape reproduction was determined by the label quite independently of the visual features of the stimulus. Inspection of Figure 2 also shows that the paralog condition is independent of scoring method and serves as a baseline index of clustering when the label training has no relevance to the categorization scheme. 


\section{DISCUSSION}

The results are as predicted; language in the form of labeling exerts a strong influence on the organization and retrieval of nonverbal representations. Both of the category name conditions recalled more shapes than did the paralog condition. These data demonstrate the influence of labeling on visual memory. They also have bearing on Ellis's conceptual coding hypothesis which maintains that labels exert their influence on visual memory by focusing attention on distinctive stimulus features. Ellis finds labels to be effective only when they are representative of the shapes they encode. The present assignment of labels to shapes was completely arbitrary and without relevance to the characteristics of the shapes. Therefore, it appears that the attentional-encoding effects of labeling, which Ellis has documented in recognition, are not the only influences of labeling operative in reproduction. The present data are better explained in terms of organization and retrieval effects of language.

However, the quantitative effects of labeling are not nearly as compelling as the qualitative influence. Assigning names to the visual stimuli strongly determined the order in which they were drawn. The implications are clear. When we learn to partition and label our perceptual experience, the qualitative way in which we remember and think about our experience is also determined. The assignment of a name to an object or experience establishes a context, and so determines the circumstances of recall. These influences are certainly one manifestation of the Whorfian hypothesis which suggests: "We dissect nature along lines laid down by our native languages. The categories and types that we isolate from the world of phenomena we do not find there because they stare every observer in the face; on the contrary, the world is presented in a kaleidoscopic flux of impressions which has to be organized by our minds-and this means largely by the linguistic systems in our minds. We cut nature up, organize it into concepts, and ascribe significance as we do largely because we are parties to an agreement to organize it in this way-an agreement that holds throughout our speech community and is codified in the patterns of our language." (Whorf, in Carroll, 1956, p. 213).

It is of course tempting to denigrate the importance of such statements by claiming, "Yes, labels might influence our remembrance of experience, but experience determines our labeling." Such an argument suggests that language is, afterall, irrelevant to our conception of the world in that it simply reflects experience. But is it so; are labels strictly reflective of the perceptual experience? Consider our labeling of hawks vs. doves and girls vs. women. These labels bring more to an experience than a simple description. They activate an entire world of context; replete with rich associations, and therefore bias. Nor are the labels

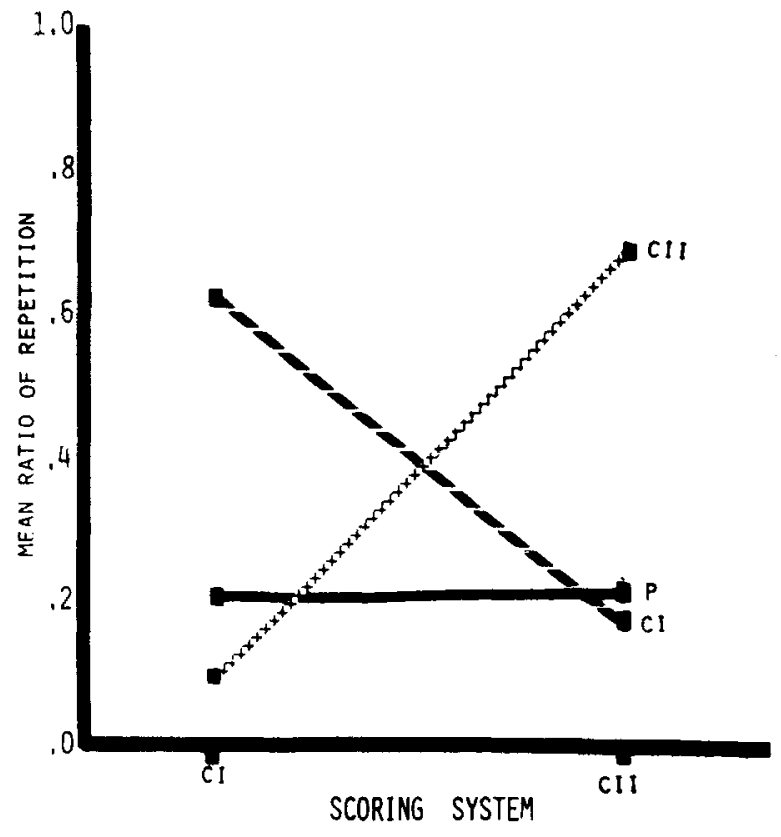

Figure 2. Clustering index as a function of scoring method (see text) and name training condition (CI, CII, paralog).

strictly determined by stimulus properties, but are applied arbitrarily (at best) or perhaps with full psychological intent. Therefore, when the present experiment demonstrates the influence of label properties independent of stimulus characteristics, it documents experimentally the power of language to influence nonverbal thought.

\section{REFERENCE NOTES}

1. Nicosia, G. J. Verbal labels and recall of visual forms. Unpublished masters thesis, Rutgers University, 1973.

2. Santa, J. L. Redintegrative recognition of shapes facilitated by name learning. Unpublished masters thesis, Purdue University, 1969.

3. Santa, J. L. The effects of verbal coding on recognition memory. Unpublished doctcral dissertation, Purdue University, 1970.

\section{REFERENCES}

Battig, W. F., \& Montague, W. E. Category norms for verbal items in 56 categories: A replication and extension of the Connecticut category norms. Joumal of Experimental Psy chology, 1969, 80 (3, Part 2).

Brown, R. W., \& Lenneberg, E. H. A study in language and cognition. Journal of Abnormal and Social Psychology, 1954,

Carmichael, L., Hogan, H. P. \& Walter, A. A. An experimental study of the effect of language on the reproduction of visually perceived forms. Journal of Experimental Psychology, 1932,
15, 73-86.

Carroll, J. E. (Ed.), Language, thought, and reality: Selected writings of Benjamin Lee Whorf. Cambridge, Mass: M. I. T. Press, 1956.

Daniel, T. C., \& Ellis, H. C. Stimulus codability and long-term recognition memory for visual form. Joumal of Experimental Psychology, 1972, 93, 8389.

Ellis, H. C. Transfer of stimulus predifferentiation to shape recognition and identification learning: Role of properties of verbal labels. Joumal of Experimental Psychology, 1968, 78,
$401-409$.

Ellis, H. C. Verbal processes in the encoding of visual pattern information: An approach to language, perception, and 
memory. In M. E. Meyer (Ed.). Third Western Symposium on Learning: Cognitive learning. Bellingham: Western Washington State College, 1972.

Ellis, H. C., \& Daniel, T. C. Verbal processes in long-term stimulus-recognition memory. Journal of Experimental Psychology, 1971, 90, 18-26.

Ellis, H. C., \& Muller, D. G. Transfer in perceptual learning following stimulus predifferentiation. Joumal of Experimental Psychology, 1964, 68, 388-395.

Frost, N. Clustering by visual shape in the free recall of pictorial stimuli. Joumal of Experimental Psychology, 1971, 88. 409-413.

Frost, N. Encoding and retrieval in visual memory tasks. Journal of Experimental Psychology, 1972, 95, $317-326$.

Funkhouser, G. R. Effects of differential encoding on recall. Journal of Verbal Learning and Verbal Behavior, 1968. 7. 1016-1023.

Lantz, D. L., \& Stefflre, V. Language and cognition revisited. Journal of Abnormal and Social Psychology. 1964. 69. $472 \cdot 481$.

Lenneberg. E. H. Biological foundations of language. New York: Wiley, 1967.

Noble, C. E. An analysis of meaning. Psychological Review, $1952,59,421$.

Rosch, E. H. Natural Categories. Cognitive Psychology. 1973. 4, 328-350.

Santa, J. L., \& Ranken, H. B. Language and memory: Redintegrative memory for shapes facilitated by naming. Psy chonomic Science, 1968, 13, 109-110.

Santa, J. L., \& Ranken, H. B. Effects of verbal coding on recognition memory. Joumal of Experimental Psychology. $1972,93,268-278$.

Slobin, D. I. Psycholinguistics. Glenview, lll: Scott, Foresman, 1971 .
Tversky, B. Eye fixations in prediction of recognition and recall. Memory and Cognition, 1974, 2, 275-278.

\section{NOTES}

1. This multiple testing procedure means that the test scores are not independent; the first test could influence the second. However, this dependence is not of particular concern unless However, this dependence is not of there is evidence that the presence of a test will interact with suspect such an interaction. Moreover, we feel that the use of both immediate and delayed tests is a reasonable strategy for both immediate and delayed interval. When subjects are given a list of items to be remembered, they expect to be tested. If they are given an immediate test, they leave with a sense of completion and are unlikely to consciously rehearse for a later test. On the other hand, if subjects are not asked to recall the items immediately, they leave expecting to be tested at some later time; they are likely to rehearse and test themselves. Consequently, the amount of rehearsal and testing is probably under better control with an immediate test procedure than when retention interval effects are measured with independent groups.

2 . Recall of the labels might depend on previous reproduction of the shapes. The test was included simply to ascertain the accessibility of the labels at the end of the final reproduction period.

(Received for publication July 26, 1974; revision accepted December $7,1974$. 